\title{
ArcheoSciences
}

Revue d'archéométrie

\section{Application des micro-spectrométries infrarouge et Raman à l'étude des processus diagénétiques altérant les ossements paléolithiques}

Application of Infrared and Raman Micro-Spectroscopy to the Study of Diagenetic Processes Altering Palaeolithic Bones

Matthieu Lebon, Katharina Müller, Ludovic Bellot-Gurlet, Céline Paris et Ina Reiche

\section{OpenEdition}

Journals

Édition électronique

URL : https://journals.openedition.org/archeosciences/3114

DOI : 10.4000/archeosciences.3114

ISBN : 978-2-7535-1849-0

ISSN : 2104-3728

Éditeur

Presses universitaires de Rennes

Édition imprimée

Date de publication : 30 avril 2011

Pagination : 179-190

ISBN : 978-2-7535-1847-6

ISSN : 1960-1360

Référence électronique

Matthieu Lebon, Katharina Müller, Ludovic Bellot-Gurlet, Céline Paris et Ina Reiche, « Application des micro-spectrométries infrarouge et Raman à l'étude des processus diagénétiques altérant les ossements paléolithiques », ArcheoSciences [En ligne], 35 | 2011, mis en ligne le 30 avril 2013, consulté le 28 février 2022. URL : http://journals.openedition.org/archeosciences/3114; DOI : https://doi.org/ 10.4000/archeosciences.3114 


\title{
Application des micro-spectrométries infrarouge et Raman à l'étude des processus diagénétiques altérant les ossements paléolithiques
}

\author{
Application of Infrared and Raman Micro-Spectroscopy to the Study \\ of Diagenetic Processes Altering Palaeolithic Bones
}

\author{
Matthieu Lebon*, **, Katharina Müller ${ }^{* *}$, Ludovic Bellot-Gurlet ${ }^{* * *}$, \\ Céline Paris ${ }^{* * *}$ et Ina ReICHE**
}

\begin{abstract}
Résumé : Différents processus post-mortem peuvent altérer les ossements et limiter l'utilisation de leur composition élémentaire et isotopique comme marqueurs paléo-environnementaux/climatiques, paléo-alimentaires ou encore géochronologiques. De part leur structure complexe et hiérarchisée, les ossements in vivo sont des tissus hétérogènes. La prise en compte de cette hétérogénéité, renforcée au cours de la fossilisation, nécessite l'utilisation de techniques d'analyses adaptées permettant l'étude des variations de structure et de compositions à micro-échelle. De précédents travaux ont montré que des techniques de micro-spectrométrie vibrationnelle telle que la micro-spectrométrie infrarouge pouvait permettre de caractériser les modifications induites par les processus diagénétiques et de déterminer les états de préservation des échantillons fossiles. Dans cette étude, des indices permettant de déterminer la composition d'ossements fossiles ont été acquis en spectrométrie infrarouge et Raman, ceci dans le but de comparer les informations fournies par ces deux techniques. Les micro-spectrométries IR et Raman ont ensuite été appliquées à des échantillons fossiles préparés sous forme de coupes minces ou de lames épaisses. Ces premiers résultats de cartographie obtenus par micro-spectrométrie Raman permettent de mettre en évidence l'apport de cette technique à la compréhension des processus diagénétiques en permettant de déterminer la répartition des principaux composés des ossements fossiles (teneur en collagène, en carbonates, cristallinité de la phase minérale, etc.).
\end{abstract}

\begin{abstract}
Various post-mortem processes can alter bone materials and then limit the use of their elemental or isotopic composition as markers for palaeoenvironmental/climatic, paleodiet, or geochronological studies. Due to their complex and hierarchical structure, in vivo bones correspond to a highly heterogeneous tissue. The study of this heterogeneity, reinforced during fossilization processes, requires the use of adapted analytical techniques to investigate the variation of composition at microscale. Previous works have demonstrated that vibrational spectroscopic techniques like infrared micro-spectroscopy can be used to characterize the modification of composition induced by diagenetic processes and to determine the preservation state of the fossil samples. In this study, some spectral parameters, used to determine the composition of fossil bones, were measured by both infrared and Raman spectroscopy in order to compare the information supplied by these two techniques. Infrared and Raman micro-spectroscopy were then applied to fossil samples prepared as thin sections or polished thick sections. These first results of chemical mapping obtained on fossil bones by Raman micro-spectroscopy are in good agreement with the data obtained by FTIR micro-spectroscopy. These results demonstrated the capacity of this method for the understanding of diagenetic processes by monitoring the main components of fossil bone tissues (carbonates and collagen content, mineral crystallinity, etc.).
\end{abstract}

Mots clés : ossements fossiles, altération diagénétique, micro-spectrométrie infrarouge et Raman, Cristallinité, Phase minérale et organique.

Keywords: fossil bones, diagenetic alterations, infrared and Raman microspectroscopy, Crystallinity, Organic and mineral phases.

* UMR - 7194 CNRS, Département de Préhistoire, Muséum national d'Histoire naturelle. (lebon@mnhn.fr)

**UMR 171 - CNRS, Laboratoire du Centre de Recherche et de Restauration des Musées de France. (katharina.mueller@culture.gouv.fr) (ina.reiche@ culture.gouv.fr)

***UMR 7075 - CNRS/UPMC, Laboratoire de Dynamique, Interactions et Réactivité (LADIR). (ludovic.bellot-gurlet@upmc.fr) (celine.paris@upmc.fr) 


\section{INTRODUCTION}

De nombreux processus sont susceptibles de modifier et d'altérer les ossements après la mort. Ces processus, dits taphonomiques, peuvent intervenir dès les premières phases de putréfaction des tissus mous et se prolonger dans l'environnement sédimentaire tout au long de l'enfouissement (diagenèse), sous l'effet combiné de facteurs biologiques et géochimiques (Collins et al., 2002; Hedges, 2002). L'étude de ces processus taphonomiques, et de leurs effets sur les assemblages osseux en contexte archéologique et paléontologique, peut constituer une source d'information permettant de reconstituer l'ensemble des évènements intervenus entre la mort de l'animal et la découverte de restes squelettiques, c'est-à-dire de déterminer leur histoire taphonomique. Les processus bio/physico-chimiques peuvent également entrainer des changements profonds de la structure et de la composition des ossements, et modifier ou supprimer certains signaux biomoléculaires, élémentaires ou isotopiques (Hedges et al., 1995; Hedges, 2002; Trueman et al., 2004; Trueman et al., 2008). De nombreux travaux ont ainsi démontré que les altérations diagénétiques pouvaient limiter l'utilisation des ossements fossiles comme source d'information dans le cadre des reconstitutions paléo-climatiques/environnementales, de l'étude des régimes alimentaires, des analyses paléogénétiques ou encore des études géochronologiques (Lee-Thorp, 2002; Lee-Thorp et Sponheimer, 2003; Bocherens et al., 2008).

Différentes méthodes ont ainsi été développées afin de caractériser les altérations diagénétiques, d'évaluer l'état de préservation des ossements fossiles et de comprendre les processus mis en œuvre. Cependant, très peu de données sont disponibles à ce jour sur les variations de composition ou de structure des phases organique ou minérale des ossements fossiles à micro-échelle. Seuls quelques travaux ont permis l'obtention de données sur la composition élémentaire par analyse par faisceau d'ion (PIXE/PIGE) ou par spectrométrie de masse (Reiche et al., 1999; Goodwin et al., 2007, Gaschen et al., 2008; Olivares et al., 2008, Hinz et Kohn, 2010), ainsi que sur la préservation de la trame organique par Microscopie Électronique à Transmission (Reiche et al., ce volume, Chadefaux, 2009). Seuls des paramètres structuraux tels que la taille et l'épaisseur moyenne des cristaux de la phase minérale de l'os ont également pu être obtenus in situ par diffusion des rayons $\mathrm{X}$ aux petits angles (SAXS) (Wess et al., 2002). D'autres techniques peuvent permettre d'accéder à des informations structurales et de composition à micro-échelle. C'est notamment le cas de la micro-spectrométrie infrarouge (IR-TF) qui présente l'avantage de fournir des informations résolues spatialement, à la fois pour les phases organique et minérale de l'os. Cette technique a ainsi été largement utilisée dans le domaine biomédical pour l'étude des échantillons modernes (Paschalis et al., 1996, Boskey et Mendelsohn, 2005, Boskey et PleshkoCamacho, 2007). La micro-spectrométrie IR a également été appliquée récemment afin de caractériser à micro-échelle les modifications de composition induites par les processus diagénétiques (Reiche et al., 2010; Lebon et al., 2011). Ce type d'analyses par spectrométries vibrationnelles s'est révélé pertinent afin d'améliorer notre compréhension des phénomènes d'altération diagénétique et de mieux évaluer l'état de conservation des échantillons.

Une autre spectrométrie vibrationnelle, la micro-spectrométrie Raman a été appliquée dans le domaine biomédical afin de cartographier les variations de composition au niveau des structures histologiques des ossements modernes (Timlin et al., 1999; Kazanci et al., 2007; Goodyear et al., 2009; Gamsjaeger et al., 2010; Shen et al., 2010). Cette technique a aussi été utilisée afin de déterminer localement l'état de préservation d'échantillons fossiles ou la conservation de matériel osseux dans les collections muséales (Pucéat et al., 2004; Edwards et al., 2005; Thomas et al., 2007; Le Blond et al., 2009).

Le but de cette étude est de présenter des premiers tests de cartographies réalisées par micro-spectrométrie Raman afin de caractériser les modifications de composition à l'échelle histologique de l'os pendant la diagenèse. Ces cartographies Raman seront comparées à celles enregistrées en spectrométrie IR-TF sur des échantillons similaires. Il sera ainsi possible d'évaluer le potentiel de cette technique pour l'étude in situ des processus d'altérations diagénétiques.

Afin de caractériser l'état de préservation des échantillons présentés ci-dessous, plusieurs paramètres spectraux permettant de caractériser les phases organique et minérale ont été mesurés à la fois en spectrométrie Raman et infrarouge. Ces paramètres spectraux permettront notamment de caractériser la proportion relative de collagène, de carbonates, ainsi que la cristallinité de la phase minérale des échantillons fossiles. Avant d'être appliquées à un échantillon archéologique préparé sous forme de coupes minces, les résultats fournis par ces deux types de spectrométries ont tout d'abord été comparés pour des échantillons sous forme de poudre d'ossements chauffés et non chauffés retrouvés en contexte archéologiques. En effet, différents travaux ont mis en évidence une perte de la matière organique et des carbonates au cours de la chauffe. La perte de ces deux composés s'accompagne de la recristallisation de la phase minérale à haute température $\left(>650^{\circ} \mathrm{C}\right)$ qui conduit à une augmentation importante de la cristallinité (Stiner et al., 1995; Hiller et al.; 2003, Lebon, 2008; Lebon et al., 2008, Piga et al., 2008; Lebon et al., 
2010). Une sélection d'échantillons présentant différents états de chauffe peut ainsi permettre d'appliquer les paramètres spectraux établis en spectrométrie IR-TF et Raman à des échantillons présentant des différences importantes de composition et de structure. Des différences de composition dues à des processus diagénétiques très distincts entre les deux sites archéologiques étudiés peuvent s'ajouter aux modifications des différences de composition induites par les états de chauffe. À travers cette étude, il sera ainsi possible de comparer les informations fournies par ces deux techniques d'analyses avant de les appliquer à des échantillons fossiles préparés sous forme de coupe mince obtenue par microtomie ou de coupes épaisses.

\section{Matériel et Méthode}

\section{Ossements fossiles préparés sous forme de poudre}

Les échantillons osseux étudiés proviennent de deux sites archéologiques pléistocènes. Il s'agit des sites de BizeTournal (Aude-France, $15 \mathrm{ka}$ [Tavoso, 1987]) et de Song Terus (Java-Indonésie, 60-80 ka [Sémah et al., 2004]). Pour chacun des sites, des échantillons chauffés et non chauffés ont été sélectionnés sur la base de critères macroscopiques tels la présence ou l'absence de traces de carbonisation ou de calcination (Lebon et al., 2010). Les échantillons non chauffés du site de Bize-Tournal sont généralement peu altérés puisqu'ils contiennent du collagène préservé. Les échantillons provenant du site de Song Terus sont quant à eux fortement altérés puisque la totalité de leur matière organique a disparue, et que leur phase minérale est partiellement recristallisée en fluorapatite (Lebon et al., 2010).

Les échantillons présentés ici ont fait l'objet d'une précédente étude exhaustive par spectrométrie IR-TF (Lebon, $e t$ al., 2010). Pour chaque échantillon, un prélèvement a été effectué en utilisant une mèche diamantée à faible vitesse de rotation pour éviter toute chauffe de l'échantillon. Une partie de cet échantillon a alors été utilisée pour la spectrométrie infrarouge, l'autre pour la spectrométrie Raman.

Les échantillons pour la spectrométrie IR-TF ont été préparés selon la méthode du pastillage. Quelques milligrammes de chaque échantillon ont été broyés dans de l'acétone afin d'obtenir une taille de grains très inférieure à $5 \mu \mathrm{m}$. Les pastilles ont ensuite été préparées en mélangeant $2,5 \mathrm{mg}$ de l'échantillon broyé complété à $1 \mathrm{~g}$ par du bromure de potassium $(\mathrm{KBr})$. Enfin, $300 \mathrm{mg}$ de ce mélange sont pesés et pressés à $11 \mathrm{t} . \mathrm{cm}^{-1}$ pendant $1,5 \mathrm{~min}$. Les données IR-TF ont alors été collectées en transmission entre 4000 et $400 \mathrm{~cm}^{-1}$ sur un spectromètre Vector 22 (Bruker) équipé d'un détecteur DGTS, par l'accumulation 64 spectres avec une résolution spectrale de $2 \mathrm{~cm}^{-1}$.

Les spectres Raman ont été collectés sur un spectromètre RFS 100/S (Bruker) utilisant comme source excitatrice une diode laser Nd : YAG (1064 nm) et un détecteur germanium refroidi à l'azote liquide. Les spectres Raman ont été enregistrés entre 3550 et $50 \mathrm{~cm}^{-1}$ avec une résolution spectrale de $4 \mathrm{~cm}^{-1}$, en accumulant de 128 à 512 scans afin d'obtenir un rapport signal/bruit satisfaisant. L'interface d'analyse macroscopique du spectromètre permet de focaliser le rayonnement laser (fixé à $100 \mathrm{MW}$ sur l'échantillon) sur une zone de 100x100 $\mu^{2}$ à la surface de l'échantillon sous forme de poudre, placé sur un miroir d'or afin d'améliorer l'intensité du signal Raman récolté par le spectromètre.

\section{Ossements fossiles préparés sous forme de coupe mince}

Les micro-spectrométries infrarouge et Raman ont été appliquées à deux échantillons préparés sous forme de coupes minces obtenues par microtomie, selon le protocole présenté ci-dessous. Il s'agit de l'échantillon ST-Ta-8 provenant du site de Song Terus et de l'échantillon BZ-O30-9 provenant du site de Bize Tournal. L'état de conservation de ces échantillons est représentatif de l'état général du matériel osseux fossile provenant de ces sites.

L'analyse d'échantillons en transmission par micro-spectrométrie IR-TF nécessite leur préparation sous forme de coupes minces d'une épaisseur de l'ordre de $2 \mu \mathrm{m}$. Cette étape n'est possible pour des échantillons fossiles altérés qu'après leur induration par de la résine (Lebon et al., 2011). De petits fragments d'échantillons $\left(0,5 \times 0,5 \times 1 \mathrm{~cm}^{3}\right)$ ont donc été déshydratés avant d'être inclus dans une résine de polyméthylméthacrylate (PMMA). Cette résine a été choisie car ses bandes les plus intenses se situent en dehors des zones d'intérêt du matériau osseux. Pour chaque échantillon, plusieurs coupes minces de $2 \mu \mathrm{m}$ d'épaisseur et d'une taille comprise entre $500 \times 500$ et $500 \times 1000 \mu \mathrm{m}^{2}$ ont été obtenues en utilisant un ultramicrotome Leica EM UC6 (plateforme IPANEMA-SOLEIL). Les analyses par microspectrométrie IR-TF ont été réalisées à la ligne SMIS du synchrotron SOLEIL (Gif-sur-Yvette, France), en utilisant un microscope Continuum couplé à un spectromètre Nicolet 5700 (ThermoNicolet). Le microscope IR-TF est équipé d'un détecteur Mercure-Cadmium-Telluride (MCT-A) collectant le rayonnement IR dans la gamme $4000-650 \mathrm{~cm}^{-1}$. Les sections fines d'ossements fossiles ont été placées sur des fenêtres $\mathrm{BaF}_{2}$ (MolTeck, $\mathrm{GmBH}$ ) et fixées à ce support par une goutte d'éthanol. En séchant, l'éthanol permet à la 
coupe d'adhérer à son support ce qui assure ainsi la planéité de la surface. Les supports $\mathrm{BaF}_{2}$ avec les échantillons ont ensuite été placés sur la plateforme motorisée du microscope afin d'être analysés en mode transmission en utilisant une ouverture de diaphragme de $12 \times 12 \mu \mathrm{m}^{2}$ et un pas de $10 \mu \mathrm{m}$ entre chaque point d'analyse. Pour chaque pixel de la zone analysée, 128 scans ont été accumulés sur la gamme spectrale 4000-400 $\mathrm{cm}^{-1}$ avec une résolution spectrale de $4 \mathrm{~cm}^{-1}$. Des backgrounds ont été réalisés dans les mêmes conditions avant chaque cartographie. Pour chaque échantillon, des zones d'au moins $100 \times 100 \mu \mathrm{m}^{2}$ ont été étudiées. Ces zones ont été choisies en dehors des parties de la coupe présentant des artéfacts de préparation (fissurations, replis, manque de planéité, etc.). L'ensemble du protocole de préparation des échantillons et du mode de traitement des données est détaillé dans Lebon et al. (2011).

Des analyses Raman ont pu être également réalisées sur ces mêmes coupes minces et des sections polies en utilisant un spectromètre Raman DXR (ThermoScientific) avec une source laser à $532 \mathrm{~nm}$. Le microscope muni d'un objectif x40 permet de focaliser le faisceau laser sur une zone d'environ $5 \mu \mathrm{m}$. Ces conditions analytiques permettent d'obtenir un bon compromis entre résolution spatiale et intensité du signal Raman. Pour chaque point d'analyse, cinq à dix spectres de 10 à $15 \mathrm{~s}$ ont été accumulés avec une résolution spectrale de $4 \mathrm{~cm}^{-1}$ afin d'obtenir un rapport signal/bruit suffisant.

\section{Ossement fossile préparé sous forme de coupe épaisse}

Un échantillon provenant du site de Song Terus a également été préparé sous forme de lame polie avant d'être étudié par micro-spectrométrie Raman. Cet échantillon (ST-Ta-3) correspond à un ossement chauffé présentant des traces de calcination avancée sur ces faces externes, tandis que la partie interne est restée carbonisée. Après la réalisation d'une section transversale à l'axe longitudinal de l'os sans aucune préparation préalable, une des faces a donc été polie afin de procéder aux analyses Raman (fig. 1c). Le polissage permet une visualisation des zones d'intérêt par microscopie optique avant d'entreprendre les analyses Raman et assure une focalisation constante du rayonnement laser au cours de l'analyse. Cet échantillon, trop fragile, n'a pas pu être préparé sous forme de coupe fine nécessaire à une analyse par micro-spectrométrie IR en transmission.

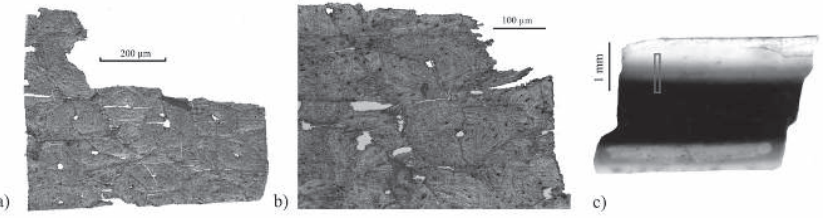

Figure 1 : (Voir planche couleur) Vue en microscopie optique de deux coupes minces obtenues pour l'échantillon ST-Ta-8, étudiées (a) en micro-spectrométrie infrarouge avec source de lumière synchrotron et (b) en micro-spectrométrie Raman. (c) Section polie de l'échantillon ST-Ta-3 étudié par spectrométrie Raman.

Figure 1: (See colour plate) Optical micrograph of two thin sections obtained for the sample ST-Ta-8, and studied by (a) infrared micro-spectrometry using synchrotron radiation and (b) Raman micro-spectrometry. (c) Polished section of the sample ST-Ta-3 studied by Raman microspectrometry.

\section{Paramètres spectraux utilisés pour la caractérisation des échantillons}

À partir des spectres obtenus en spectrométries IR-TF et Raman, différents indices ont été mesurés afin de caractériser les échantillons. Les figures $2 \mathrm{a}$ et $2 \mathrm{~b}$ présentent des spectres typiques d'un ossement fossile bien préservé provenant du site de Bize-Tournal, acquis respectivement en spectrométrie IR et Raman.

\section{Cristallinité de la phase minérale}

L'augmentation de la cristallinité de la phase minérale des ossements au cours de la diagenèse est couramment évaluée en spectrométrie IR par l'intermédiaire du «Splitting Factor " (IRSF) (Weiner et Bar-Yosef, 1990). Cet indice de cristallinité est basé sur le degré d'individualisation des deux bandes phosphates $\left(v_{4} \mathrm{PO}_{4}\right)$ situés à 565 et $605 \mathrm{~cm}^{-1}$ (fig. 2c). Plus ces deux bandes sont individualisées, plus l'IRSF et la cristallinité (taille et perfection des cristaux) sont importantes. D'autres indices de cristallinité peuvent également être mesurés en se basant sur le domaine spectral situé entre 1200 et $900 \mathrm{~cm}^{-1}$. Ce large massif spectral résulte de plusieurs composantes correspondant aux modes de vibration $v_{1}$ et $v_{3}$ des groupements phosphates (Rey et al., 1991; Gadaleta et al., 1996; Lebon et al., 2008). Plusieurs travaux ont montré que l'établissement de rapport entre des composantes attribuées aux groupements phosphates dans un environnement stœchiométrique, et des composantes attribuées aux groupements phosphates dans un environnement non stœechiométrique, pouvait permettre d'évaluer la cristallinité de la phase minérale. C'est notamment le cas des indices basés sur les rapports d'intensité des composantes $1030 \mathrm{~cm}^{-1} / 1020 \mathrm{~cm}^{-1}$ et $1060 \mathrm{~cm}^{-1} / 1075 \mathrm{~cm}^{-1}$ (Paschalis et 

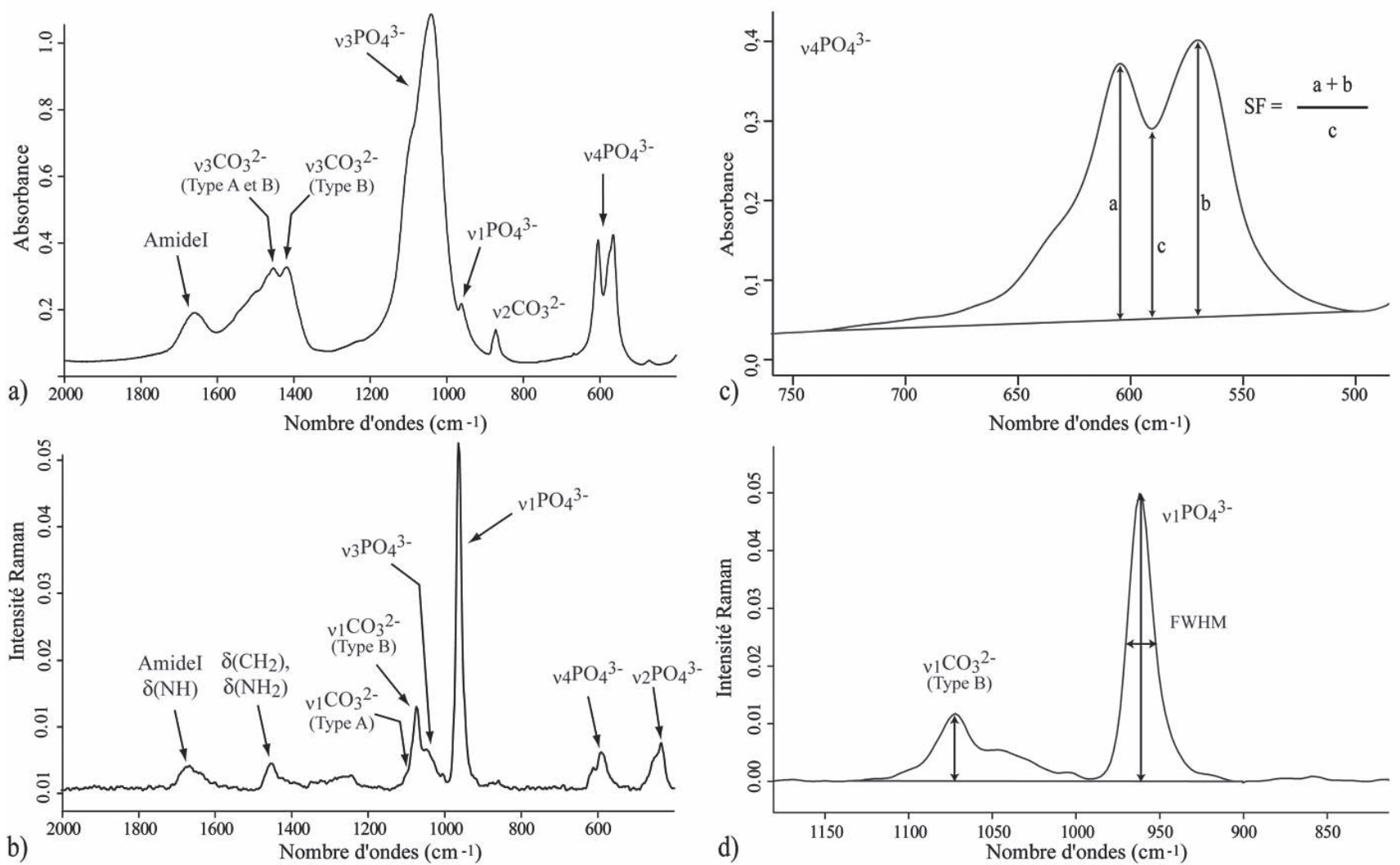

Figure 2 : Spectres typiques d'un ossement fossile (BZ-O30-17) bien préservé acquis en spectrométrie IR-TF (a) et FT-Raman (b). (c) Mode de calcul de l'indice de cristallinité en spectrométrie infrarouge ("Splitting Factor », IRSF). (d) Détail de la gamme spectrale $1200-800 \mathrm{~cm}^{-1}$ en spectrometrie Raman.

Figure 2: Typical spectra of a well preserved fossil bone (BZ-O30-17) analysed by FTIR spectrometry (a) and FT-Raman (b). Calculation mode of the crystallinity index in infrared spectrometry ("Splitting Factor", IRSF). (d) Detail of the 1200-800 cm 1 spectral range in Raman spectrometry.

al., 1996 Lebon et al., 2010). Dans le cadre de cette étude, la cristallinité des échantillons a été évaluée par l'intermédiaire de l'IRSF, ainsi que de l'indice 1060/1075. Une précédente étude a mis en évidence une bonne correspondance entre ces deux modes d'évaluation de la cristallinité pour des ossements fossiles (Lebon, et al., 2010).

En spectrométrie Raman, la cristallinité des échantillons fossiles a été évaluée par la mesure de la largeur à mi-hauteur ("Full Width at Half Maximum », FWHM) de la bande centrée vers $960 \mathrm{~cm}^{-1}$ correspondant au mode de vibration $v_{1}$ des groupements phosphates (Pucéat, et al., 2004) (fig. 2d). Différents travaux ont également montré que la position de cette bande $v_{1} \mathrm{PO}_{4}$ était susceptible de varier suite à une amélioration de l'organisation du réseau cristallin due à l'incorporation de différents ions au sein de la maille cristalline. Une augmentation de la position en fréquence de cette bande peut ainsi être utilisée afin de mettre en évidence des altérations diagénétiques et notamment un enrichissement en fluor (Thomas et al., 2007; Lebon et al., 2010).
La position de cette bande a été relevée à la fois en spectrométrie IR-TF et Raman (Figure 2a et 2b).

\section{Teneur relative en collagène et carbonates}

La préservation éventuelle de collagène au sein des échantillons fossiles, et l'évaluation de la quantité relative de collagène pour les différents échantillons fossiles constitue un paramètre important de l'évaluation de l'état de préservation. Plusieurs bandes attribuées au collagène sont identifiables sur les spectres IR-TF et Raman. La mesure de la préservation relative du collagène dans les différents échantillons peut être réalisée par le rapport d'aire ou d'intensité entre la bande amide I et la bande phosphates la plus intense, c'est-à-dire autour de $1035 \mathrm{~cm}^{-1}$ en spectrométrie IR-TF et vers $961 \mathrm{~cm}^{-1}$ en spectrométrie Raman (fig. 2a et $2 \mathrm{~b})$. De façon comparable, le rapport d'aire ou d'intensité entre bande carbonates et phosphate a permis d'évaluer la proportion relative de carbonates dans les différents échantillons (Thomas et al., 2007). La bande carbonates 
à $1415 \mathrm{~cm}^{-1}$ a été utilisée dans le cas de la spectrométrie IR-TF, tandis qu'en spectrométrie Raman, c'est la bande centrée vers $1073 \mathrm{~cm}^{-1}$ qui a été employée (fig. $2 \mathrm{a}$ et $2 \mathrm{~b}$ ). En spectrométrie IR-TF, l'intensité relative des bandes a été utilisée afin d'établir les concentrations relatives en carbonates et en collagène, alors qu'en spectrométrie Raman, la plus faible intensité de ces bandes a nécessité l'utilisation de leur aires afin d'obtenir des rapports fiables. Ces différents indices ont été mesurés suivant les modes de mesure présentés des différents travaux cités précédemment (Pucéat et al., 2004; Thomas et al., 2007; Lebon et al., 2010). Ces différents paramètres spectraux ont été calculés en utilisant le logiciel GramsAI (ThermoScientific) pour les données IR-TF et le logiciel OPUS (Bruker) en ce qui concerne les données Raman.

\section{Résultats et Discussion}

\section{Échantillons sous forme de poudre}

\section{Cristallinité}

Les valeurs de cristallinités mesurées en spectrométrie IR (IRSF) et Raman (FWHM) pour les échantillons en poudre sont présentées en figure 3a. Une augmentation de la cristallinité de la phase minérale de l'échantillon se caractérise par une réduction de la FWHM de la bande $v_{1} \mathrm{PO}_{4}$ vers 960 $\mathrm{cm}^{-1}$ en spectrométrie Raman, tandis que l'on observe une augmentation de l'IRSF en spectrométrie IR. Les échan- tillons calcinés présentent des valeurs d'IRSF supérieures à 7,5 et une FWHM de la bande $v_{1} \mathrm{PO}_{4}$ inférieure à $10 \mathrm{~cm}^{-1}$, sans qu'il soit possible d'établir de corrélation entre ces deux valeurs. En ce qui concerne les échantillons non chauffés, ils présentent des valeurs d'IRSF comprises entre 3,2 et 4,5, et des FWHM comprises entre 14,4 et 17,2 $\mathrm{cm}^{-1}$. Une corrélation entre les mesures de cristallinité obtenues en spectrométrie IR et en spectrométrie Raman pour les échantillons non chauffés $\left(\mathrm{r}^{2}=0,6, \mathrm{p}<0,01\right)$ a été observée. Pour la position de la bande $v_{1} \mathrm{PO}_{4}$ une bonne corrélation entre les mesures effectuées en spectrométrie Raman et IR a également été mise en évidence $\left(\mathrm{r}^{2}=0,77, \mathrm{p}<0,01\right)$.

\section{Teneurs en carbonates et en collagène}

L'évaluation de la proportion relative des carbonates dans la phase minérale a également pu être déterminée en utilisant le rapport des bandes carbonates et phosphates (fig. 3c). Une corrélation est observée entre les données obtenues en spectrométrie IR et Raman, mais celle-ci est essentiellement représentée par deux groupes de points distincts. Il semble donc que la mesure des teneurs en carbonates soit plus difficile en spectrométrie Raman qu'en IR-TF. Il en est de même en ce qui concerne le collagène, puisque la détection et la mesure de la bande amide n'a été possible que pour trois échantillons en spectrométrie Raman (contre six échantillons en spectrométrie IR-TF). Ces données sont pour le moment insuffisantes pour tenter d'établir une corrélation entre les résultats de quantification du collagène établie avec ces deux méthodes.

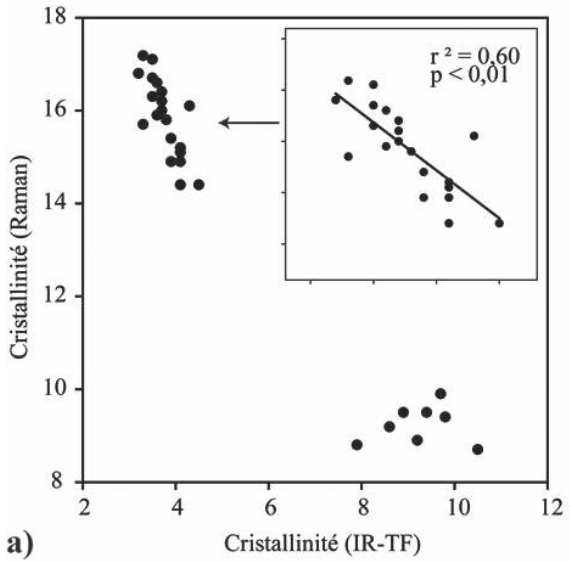

Figure 3 : Comparaison entre les données obtenues en spectrométrie Raman et IR-TF pour les valeurs de cristallinité (a), la position en fréquence de la bande $v_{1} \mathrm{PO}_{4}(\mathrm{~b})$, et de la teneur relative en carbonates (c).

Figure 3: Comparison of the data obtained by Raman and FTIR spectrometry for crystallinity values (a), $\vee_{1} P O_{4}$ band frequencies (b) and carbonates relative content (c).
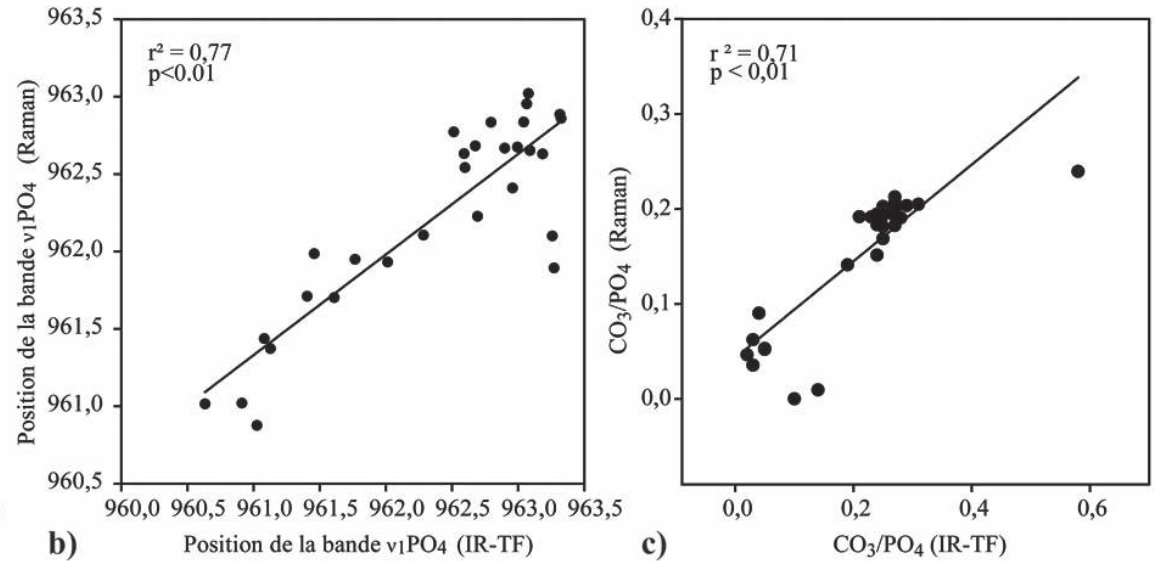


\section{Cartographie IR et Raman}

La micro-spectrométrie IR a été appliquée sur une coupe mince provenant de l'échantillon ST-Ta-8 du site de Song Terus. La figure 4 a présente une vue en microscopie optique de la zone d'analyse étudiée pour cet échantillon et les figures $4 \mathrm{~b}$ et $4 \mathrm{c}$ représentent respectivement les répartitions spatiales des teneurs en carbonates et des valeurs de la cristallinité. Aucune trace de matière organique préservée n'a pu être détectée dans cet échantillon. Une répartition très hétérogène de la teneur en carbonates et des valeurs de cristallinité au niveau de cette zone d'analyse a été observée. Ces variations importantes à micro-échelle témoignent de l'hétérogénéité des altérations diagénétiques qui interviennent à l'échelle histologique. Les valeurs de cristallinité, très supérieures à celles classiquement observées pour des échantillons modernes, mettent en évidence une importante recristallisation de la phase minérale.

Cette recristallisation est confirmée par les teneurs en carbonates assez faibles pour l'ensemble de la zone d'analyse, à l'exception de deux zones beaucoup plus riches en carbonates. Ces deux zones, entourant les porosités naturelles de l'os que constituent les canaux de Havers, présentent des teneurs en carbonates très supérieures à celles relevées dans les échantillons modernes et pourraient donc correspondre à des zones d'enrichissement en carbonates exogènes provenant des eaux d'infiltration. La teneur en carbonates au sein de la phase minérale est corrélée aux valeurs de cristallinité (fig. 5), à l'exception de quelques pixels correspondant à ces zones enrichies en carbonates en périphérie des canaux de Havers (fig. 5 , points blancs). Cette absence de corrélation entre teneurs en carbonates et valeurs de cristallinité au niveau de ces zones suggère que les carbonates ne sont pas directement incorporés à la phase minérale, mais plutôt adsorbés à la surface des cristaux ou précipités sous forme de calcite.
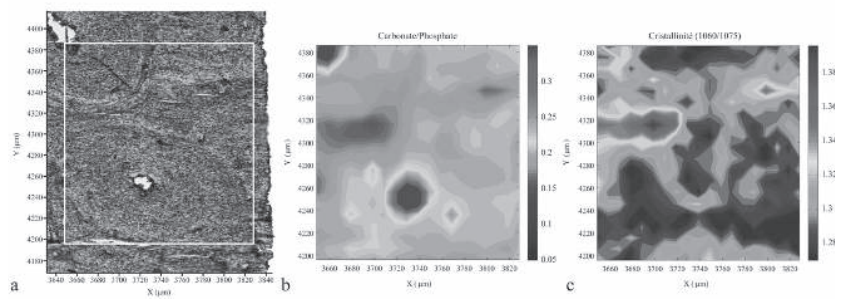

Figure 4 : (Voir planche couleur) (a) Vue optique de la zone de l'échantillon ST-Ta-8 analysée par micro-spectrométrie IR et distribution des (b) teneurs relatives en carbonates et (c) des valeurs de cristallinité dans cette même zone.

Figure 4: (See colour plate) Optical view of the area of the sample ST-Ta-8 analysed by IR micro-spectrometry and distribution of (b) the carbonate relative content, and (c) the crystallinity values in this area.

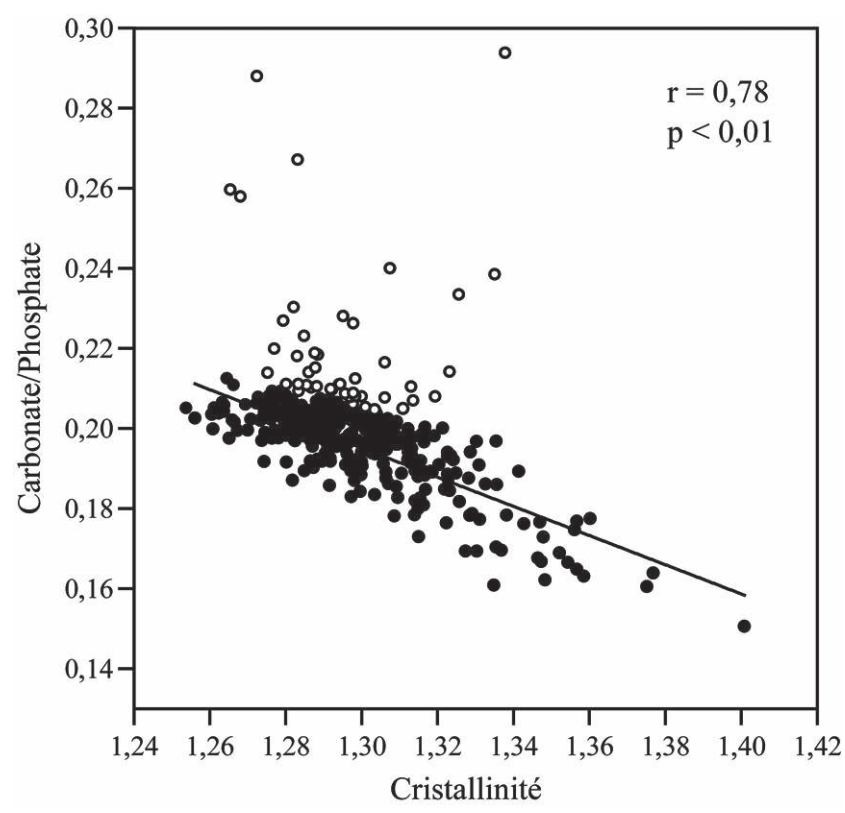

Figure 5: Relation entre les teneurs relatives en carbonates et les valeurs de cristallinité pour chacun des pixels de la zone de l'échantillon ST-Ta-8 analysée par micro-spectrométrie IR. Les points blancs correspondent aux pixels entourant les canaux de Havers visibles sur la figure 4 a.

Figure 5: Relationship between the relative content of carbonate and the crystallinity values for each pixel of the area of the sample ST-Ta-8 analysed by IR micro-spectrometry. White points correspond to the pixels surrounding the Haversian canals visible on the figure $4 a$.

Par ailleurs, il apparaît que les zones présentant des valeurs de cristallinité plus importantes se situent en périphérie, et entre, les ostéons. Des faciès similaires peuvent être observés dans ces ossements modernes où ces zones correspondent à des zones du tissu osseux beaucoup plus minéralisées (Paschalis, et al., 1996). Cependant, les valeurs de cristallinité sont ici beaucoup plus importantes que celles observées pour des ossements modernes (rapport 1060/1075 $\approx 1.12$ \pm 0.02 ; [Lebon, et al., 2011]). Il semblerait donc que les altérations diagénétiques, et notamment les recristallisations de la phase minérale, puissent dans certains cas agir de façon à conserver les faciès présents in-vivo. Le nombre limité d'échantillons traités ne permet cependant pas de conclure pour le moment sur ce type de phénomène.

Pour l'échantillon BZ-O30-9, les teneurs en carbonates sont relativement hétérogènes dans la zone d'analyse contrairement aux valeurs de cristallinité assez homogènes. Seule une zone présente une cristallinité plus élevée (FWHM plus faible). Cette zone correspond à la zone présentant un enrichissement en carbonates et semble donc plus altérée que les autres parties de la zone d'analyse. Pour cet échantillon, une 
a)

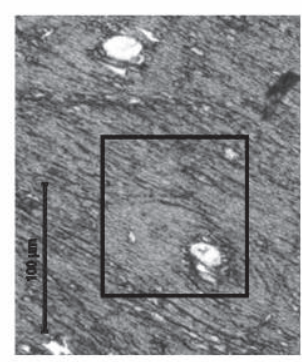

Carbonate/Phosphate

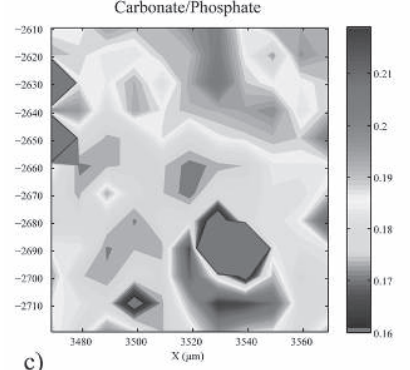

Collagène/Phosphate

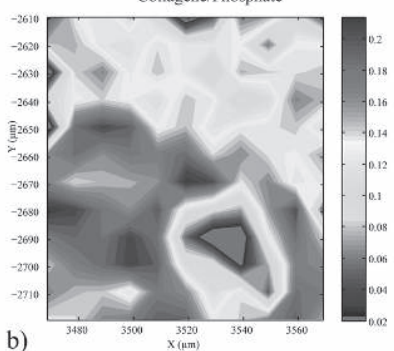

Cristallinité (FWHM)

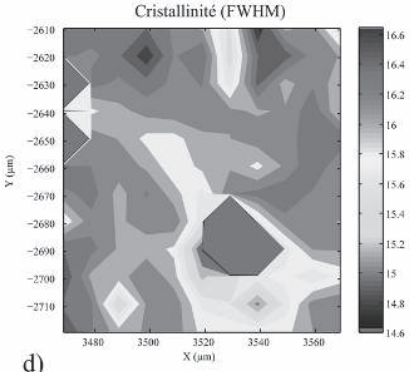

d)
Figure 6: (Voir planche couleur) a) Vue en microscopie optique de l'échantillon BZ-O30-9 analysé en spectrométrie Raman. Distribution des teneurs relatives en collagène (b), en carbonates (c), des valeurs de cristallinité (d). Les zones grisées correspondent aux zones présentant des spectres non exploitables pour la mesure de ces paramètres (présence de résine ou de calcite en quantité importante par exemple).

Figure 6: (See colour plate) a) Optical view of the thin section of the sample BZ-O30-9 analysed by Raman spectrometry. Distribution of the relative contents of collagen (b), carbonates (c), and crystallinity values (d). The shaded areas correspond to area with unusable spectra for the measurement of these parameters (presence of resin or calcite in large amount for exemple).

partie de la phase organique est préservée. La distribution du collagène est cependant très hétérogène et la partie supérieure de la zone analysée semble plus altérée, de même que la zone entourant le canal de Havers dans la partie inférieure de la cartographie Raman. Une corrélation inverse entre la distribution du collagène et celle des carbonates est observée. Bien que la mesure quantitative de ces deux composés semble possible à micro-échelle, leur détection est cependant difficile en spectrométrie Raman du fait de la faible intensité de leurs bandes correspondantes et de la faible intensité du signal Raman. Des analyses complémentaires sont en cours, notamment sur du matériel moderne, afin d'évaluer la fiabilité des mesures obtenues pour ces deux composés.

En ce qui concerne l'analyse par micro-spectrométrie Raman de l'échantillon ST-Ta-8 (fig. 7a), aucune trace de matière organique n'a été détectée ce qui confirme les résultats obtenus en micro-spectrométrie IR. Les teneurs en carbonates sont relativement faibles, à l'exception de la partie en périphérie du canal de Havers situé au centre de
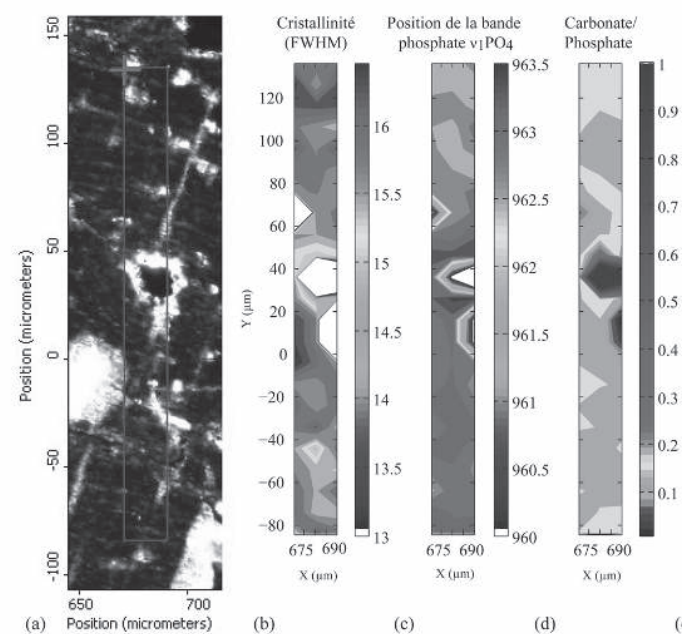

Calcite

(b)

(c)

(d)

(e)

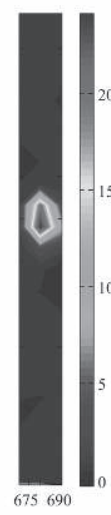

$\mathrm{x}(\mu \mathrm{m})$

Figure 7 : (Voir planche couleur) Vue optique de la zone d'analyse (a) et répartition des valeurs de cristallinité (b), des positions de la bande $v_{1} \mathrm{PO}_{4}(\mathrm{c})$, et des teneurs relatives de carbonates (d) et en calcite (e) pour l'échantillon ST-Ta-8 analysé par micro-spectrométrie Raman (532 nm). La zone analysée est également reportée en Figure 1c. Figure 7: (See colour plate) Optical view (a) and distribution of crystallinity values (b), $v_{1} P O_{4}$ bandfrequencies (c), relative contents of carbonate (d) and calcite (e) for the sample ST-Ta-8 analysed by Raman microspectrometry $(532 \mathrm{~nm})$. The analysed area is also indicated on Figure 1.

la zone d'analyse (fig. 7b). Ceci traduit une augmentation de la cristallinité liée à des phénomènes de recristallisation induits par les processus diagénétiques (fig. 7c). Des positions en fréquence importantes pour la bande correspondant au mode de vibration $v_{1}$ des groupements phosphate ont été constatées (fig. $7 \mathrm{~d}$ ). Ceci peut caractériser une amélioration de l'organisation du réseau cristallin due à différents processus diagénétiques possibles, par exemple des phénomènes de dissolution/recristallisation ainsi qu'à un enrichissement important en fluor tel qu'il a pu être mis en évidence dans une précédente étude pour les échantillons de Song Terus (Lebon, et al., 2010). Une présence importante de calcite a été mise en évidence autour du canal de Havers. La présence de calcite au niveau d'une porosité naturelle de l'os et le taux important de carbonates dans cette zone de l'échantillon témoignent d'un enrichissement en carbonates par les eaux d'infiltration. Par ailleurs, les données de micro-spectrométrie Raman montrent que cet enrichissement reste très localisé et affecte peu le tissu osseux environnant. L'ensemble de ces résultats est en accord avec les résultats obtenus en micro-spectrométrie IR et avec l'état de préservation général observé pour le matériel osseux provenant de ce site.

Une cartographie Raman a également été réalisée sur une coupe épaisse de d'un autre échantillon du site de Song 
Terus (ST-Ta-3) sur une zone à la transition entre la partie interne carbonisée et la partie externe calcinée (Figure 8a). Les teneurs relatives en carbonates apparaissent beaucoup plus importantes dans la partie carbonisée que dans la partie calcinée (fig. 8b). La FWHM de la bande $v_{1} \mathrm{PO}_{4}$ est plus faible dans la partie calcinée (fig. 8c) caractérisant ainsi une cristallinité plus importante dans cette zone de l'échantillon. Les tendances observées pour ces deux paramètres mettent en évidence la recristallisation importante de la phase minérale de l'os qui intervient lors de la calcination (température de chauffe supérieure à $650^{\circ} \mathrm{C}$ ). Les variations spatiales de
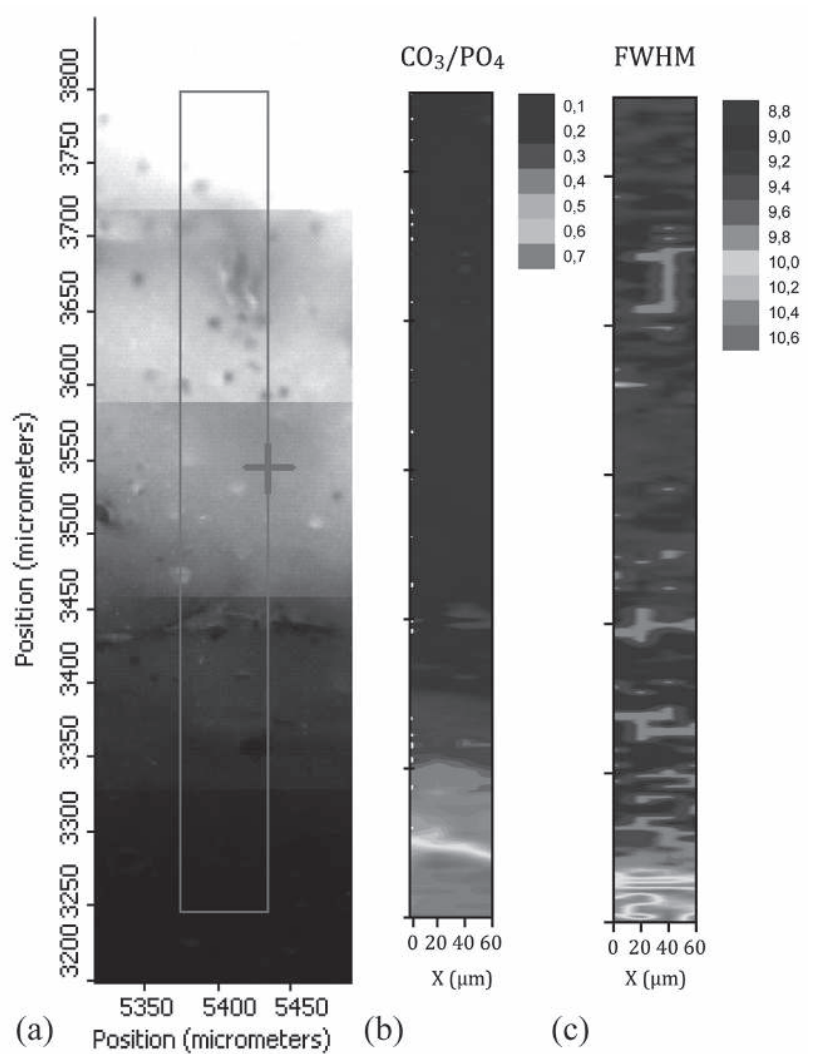

Figure 8 : (Voir planche couleur) Vue optique de la zone d'analyse (a) et distribution des teneurs relatives en carbonates (b) et des valeurs de cristallinité (c) pour l'échantillon ST-Ta-3 analysé par micro-spectrométrie Raman $(532 \mathrm{~nm})$. Le gradient de composition induit par la chauffe est clairement identifiable par l'étude de ces deux composés. La zone analysée est également reportée en Figure 1a. Pour cette zone d'analyse, un point d'analyse a été réalisé tout les $5 \mu \mathrm{m}$ en $\mathrm{X}$ et tout les $10 \mu \mathrm{m}$ en $\mathrm{Y}$.

Figure 8: (See colour plate) Optical view of the analysed area (a) and distribution of the carbonate relative contents (b) and crystallinity values (c) for the sample ST-Ta-3 analysed by Raman micro-spectrometry (532 $\mathrm{nm}$ ). The composition gradient induced by heating is clearly visible by the distribution of these two compounds. The analysed area is also indicated on Figure 1a. For this area, a spectra was acquired every $5 \mu \mathrm{m}$ in $X$ and $10 \mu \mathrm{m}$ in $Y$. composition observées pour cet échantillon correspondent donc bien aux tendances attendues compte tenu de l'état de chauffe de cet échantillon.

\section{Apport de l'imagerie infrarouge et Raman à l'étude des processus diagénétiques}

Les résultats présentés précédemment ont mis en évidence l'existence d'une hétérogénéité importante de la composition des ossements fossiles à micro-échelle. Une telle hétérogénéité nécessite la mise en place de protocoles analytiques adaptés afin d'étudier les processus de fossilisation et d'évaluer les états de préservation à micro-échelle.

Les spectrométries IR et Raman permettent une caractérisation de l'état d'altération des échantillons. Les deux techniques analytiques offrent la possibilité de déterminer les caractéristiques de la phase minérale de l'os telles que sa teneur en carbonates, le degré d'organisation du réseau cristallin, ou encore sa cristallinité qui constitue l'un des paramètres d'évaluation de l'état de préservation les plus utilisés.

En spectrométrie IR, l'évaluation de la teneur en carbonates et en collagène est relativement aisée compte tenu du bon rapport signal/bruit, même lorsque ces composés sont présents en faible concentration. Pour les échantillons analysés en spectrométrie Raman, la faible intensité des bandes correspondant au collagène et aux carbonates, et le faible rapport signal/bruit rend leur détection moins aisée et la précision des mesures devra être confirmée par des analyses complémentaires.

La micro-spectrométrie IR offre une résolution spatiale pouvant atteindre une dizaine de micromètres lorsque l'on souhaite obtenir des données sur la gamme spectrale 2000 $650 \mathrm{~cm}^{-1}$ nécessaire à l'étude des propriétés de l'os. Cette résolution est adaptée à l'étude des structures histologiques de l'os. L'obtention d'une telle résolution spatiale nécessite cependant l'utilisation de sources de lumière synchrotron, dont le temps d'accès est limité. L'obtention d'une résolution d'une résolution de l'ordre de 20 à $10 \mu \mathrm{m}$ est cependant possible en utilisant des systèmes d'imagerie de laboratoire. La spectrométrie Raman offre en général une meilleure résolution spatiale $(<5 \mu \mathrm{m})$. Cependant, les temps d'acquisition des spectres sont généralement beaucoup plus longs qu'en spectrométrie IR, ce qui ne permet pas la cartographie de zones de taille importante. L'usage de cette technique semble donc réservé à l'étude de zones d'intérêt particulières de taille restreintes avec une résolution spatiale importante.

Par ailleurs, ces deux techniques peuvent également s'avérer complémentaires pour la détection de certaines phases minérales exogènes, c'est notamment le cas de la calcite, 
difficilement identifiable en micro-spectrométrie IR mais facilement caractérisable en micro-spectrométrie Raman.

La micro-spectrométrie Raman présente un avantage majeur sur la micro-spectrométrie IR en ce qui concerne la préparation des échantillons. Le protocole d'analyse établi en micro-spectrométrie IR nécessite de préparer les échantillons sont forme de coupes minces. Le temps nécessaire pour la préparation des échantillons (de l'ordre de plusieurs jours) constitue un facteur limitant, et la taille des coupes obtenues reste limitée et difficilement contrôlable. En ce qui concerne la spectrométrie Raman, elle est applicable directement sur des surfaces polies ce qui minimise le temps de préparation et permet d'accéder à des zones d'investigation plus larges. Il pourrait alors être possible de combiner cette approche avec des analyses par micro-spectrométrie Infrarouge en mode réflexion ou en réflexion totale atténuée (ATR).

\section{Conclusion}

L'étude des processus d'altérations diagénétiques des matériaux osseux constitue un thème important de recherche depuis de nombreuses années. Ces études se sont longtemps consacrées à l'analyse de fractions globale d'échantillons, ce qui ne permet pas de prendre en compte l'hétérogénéité de la composition de l'os in vivo, de même que l'hétérogénéité des altérations induites par les processus de fossilisation. Les développements techniques réalisés ces dernières années permettent désormais de s'affranchir des difficultés analytiques et de préparation des échantillons pour permettre une étude de ces processus diagénétiques à l'échelle histologique du tissu osseux.

Dans cette étude, nous avons montré que les micro-spectrométries IR et la micro-spectrométrie Raman pouvaient permettre d'obtenir des informations résolues spatialement sur la composition moléculaire et la structure des différents constituants minéraux des ossements fossiles. Ces deux techniques peuvent ainsi permettre de caractériser différents états d'altérations, depuis les premiers stades d'altération de l'os (perte du collagène), jusqu'aux modifications plus profondes de la phase minérale (recristallisation de la phase minérale notamment).

Le protocole analytique présenté dans cette étude pourra également constituer un apport important dans le cadre de la sélection des échantillons, ou des parties d'échantillons, susceptibles de fournir des informations d'ordre paléo-isotopiques, paléo-génétiques ou encore géochronologiques.

À travers les premiers résultats présentés dans cette étude, il est possible d'entrevoir les possibilités offertes par ces deux techniques. Cependant, en raison du faible nombre d'échan- tillons étudiés jusqu'ici, il s’avère difficile de déterminer avec certitude les processus ayant conduit à la formation des facies d'altération observés. Afin d'améliorer notre compréhension des processus diagénétiques, ces deux techniques complémentaires devront être appliquées à un nombre plus important d'ossements fossiles provenant de différents contextes archéologiques et paléontologiques, afin de prendre en compte des paramètres tels que l'environnement sédimentaire, le contexte climatique et l'âge des échantillons considérés. L'étude d'un corpus plus large d'échantillons préparés sous forme de coupes polies est ainsi en cours par spectrométrie Raman. Il sera également nécessaire d'améliorer les référentiels modernes permettant de prendre en compte les différences de composition in vivo liées à l'espèce et à l'âge des individus. Ces études futures permettront ainsi de mieux appréhender les processus d'altération diagénétique et leurs effets sur le potentiel informatif de la composition des ossements fossiles.

\section{Remerciements}

Nous remercions Sophie Berland, Laurianne Robinet, MarieAngelique Languille, William Josse et Danielle Jaillard pour leurs conseils et leur aide pour la préparation des échantillons et pendant les expériences. Les auteurs remercient la plateforme IPANEMA et le synchrotron SOLEIL (projet 20990319), plus particulièrement Loüc Bertrand, Christophe Sandt et Paul Dumas, pour leur aide dans la préparation et la réalisation de ce projet. Cette étude a été réalisée dans le cadre du projet ANR07-JCJC-ArBoCo 2007-2010 ("Improvement of conservation methods of archaeological bone material ") et du programme PNRCC-OsIRIS 2010-2012 ("Potentiel informatif des ossements chauffés en contexte archéologique : Etude à micro-échelle des aspects cristallographiques, élémentaires et isotopiques") financé par le ministère de la Culture et de la Communication. Enfin, nous remercions les relecteurs de cet article pour leurs remarques et leur contribution à l'amélioration de ce manuscrit.

\section{Bibliographie}

Bocherens, H., Drucker, D.G., Billiou, D., Geneste, J.-M. et Kervazo, B., 2008 - Grotte Chauvet (Ardèche, France): A „natural experiment" for bone diagenesis in karstic context, Palaeogeography, Palaeoclimatology, Palaeoecology 266, p. 220226.

Boskey, A. et Pleshro-Camacho, N., 2007 - FT-IR imaging of native and tissue-engineered bone and cartilage, Biomaterials 28, p. 2465-2478. 
Boskey, A. L. et Mendelsohn, R., 2005 - Infrared spectroscopic characterization of mineralized tissues, Vibrational Spectroscopy 38, p. 107-114.

Chadefaux, C., 2009 - Établissement d'une nouvelle stratégie analytique multiéchelle de détermination de l'état de conservation des os et bois de cervidés archéologiques, UPMC, Paris.

Collins, M. J., Nielsen-Marsh, C. M., Hiller, J., Smith, C.I., Roberts, J. P., Prigodich, R. V., Wess, T. J., Csapo, J., Millard, A. R. et Turner-Walker, G., 2002 - The Survival of Organic Matter in Bone : a Review, Archaeometry 44, p. 383394.

Edwards, H., Jorge Villar, S., Nik Hassan, N., Arya, N., O’Connor, S. et Charlton, D., 2005. Ancient biodeterioration: an FT-Raman spectroscopic study of mammoth and elephant ivory, Analytical and Bioanalytical Chemistry 383, 713-720.

Gadaleta, S. J., Paschalis, E. P., Betts, F., Mendelsohn, R. et Boskey, A. L., 1996 - Fourier Transform Infrared Spectroscopy of the Solution-Mediated Conversion of Amorphous Calcium Phosphates to Hydroxyapatite : New Correlations Between X-Ray Diffraction and Infrared Data, Calcif Tissue Int 58, p. 9-16.

Gamsjaeger, S., Masic, A., Roschger, P., Kazanci, M., Dunlop, J. W. C., Klaushofer, K., Paschalis, E. P. et Fratzl, P., 2010 - Cortical bone composition and orientation as a function of animal and tissue age in mice by Raman spectroscopy, Bone 47, p. 392-399.

Gaschen, A. A. M., Döbeli, M., MarkwitZ, A., Barry, B., UlrichBoCHSLER, S. et KRÄHENBÜHL, U., 2008 - Restrictions on fluorine depth profiling for exposure age dating in archaeological bones, Journal of Archaeological Science 35, p. 535-552.

Goodwin, M. B., Grant, P. G., Bench, G. et Holroyd, P. A., 2007 - Elemental composition and diagenetic alteration of dinosaur bone: Distinguishing micron-scale spatial and compositional heterogeneity using PIXE, Palaeogeography, Palaeoclimatology, Palaeoecology 253, p. 458-476.

Goodyear, S. R., Gibson, I. R., Skakle, J. M. S., Wells, R. P. K. et Aspden, R. M., 2009 - A comparison of cortical and trabecular bone from C57 Black 6 mice using Raman spectroscopy, Bone 44, p. 899-907.

Hedges, R. E. M., 2002 - Bone Diagenesis : An Overview of Processes, Archaeometry 44-3, p. 319-328.

Hedges, R. E. M., Millard, A. R. et Pike, A. W. G., 1995 Measurements and Relationships of diagenetic Alteration of Bone from Three Archaeological Sites, Journal of Archaeological Science 22, p. 201-209.

Hiller, J. C., Thompson, T. H. U., Evison, M. P., Chamberlain, A. T. et Wess, T. J., 2003 - Bone mineral change during experimental heating : an X-Ray scattering investigation, Biomaterials 24, p. 5091-5097.
Hinz, E. A. et KoHn, M. J., 2010 - The effect of tissue structure and soil chemistry on trace element uptake in fossils, Geochimica et Cosmochimica Acta 74, p. 3213-3231.

Kazanci, M., Wagner, H. D., Manjubala, N. I., Gupta, H. S., Paschalis, E., Roschger et P., Fratzl, P., 2007 - Raman imaging of two orthogonal planes within cortical bone, Bone 41, p. 456-461.

Le Blond, S., Guilminot, E., Lemoine, G., Huet, N. et Mevellec, J.-Y., 2009 - FT-Raman spectroscopy: A positive means of evaluating the impact of whale bone preservation treatment, Vibrational Spectroscopy 51, p. 156-161.

Lebon, M., 2008 - Caractérisation par Spectroscopie Infrarouge à Transformée de Fourier des ossements chauffés en contexte archéologique - Comparaison entre référentiel moderne et matériel archéologique, Implication diagénétique, Département de Préhistoire, Muséum national d'Histoire naturelle, Paris, p. 339.

Lebon, M., Müller, K., Bahain, J. J., Fröhlich, F., Falguères, C., Bertrand, L., Sandt, C. et Reiche, I., 2011 - Imaging fossil bone alterations at the microscale by SR-FTIR microspectroscopy, Journal of Analytical Atomic Spectrometry 26, p. 922-929.

Lebon, M., Reiche, I., Bahain, J. J., Chadefaux, C., Moigne, A. M., Fröhlich, F., SÉmah, F., Schwarcz, H. P. et Falguères, C., 2010 - New parameters for the characterization of diagenetic alterations and heat-induced changes of fossil bone mineral using Fourier transform infrared spectrometry, Journal of Archaeological Science 37, p. 2265-2276.

Lebon, M., Reiche, I., Fröhlich, F., Bahain, J. J. et Falguères, C., 2008 - Characterization of archaeological burnt bones: contribution of a new analytical protocol based on derivative FTIR spectroscopy and curve fitting of the $v_{1} v_{3} \mathrm{PO}_{4}$ domain, Analytical and Bioanalytical Chemistry 392, p. 1479-1488.

LeE-Thorp, J., 2002 - Two decades of progress towards understanding fossilization processes and isotopic signals in calcified tissue minerals, Archaeometry 44, p. 435-446.

LeE-Thorp, J. et Sponheimer, M., 2003 - Three case studies used to reassess the reliability of fossil bone and enamel isotope signals for paleodietary studies, Journal of Anthropological Archaeology 22, p. 208-216.

Olivares, M., Etxebarria, N., Arana, G., Castro, K., Murelaga, X. et Berreteaga, A., 2008 - Multielement $\mu$-ED-XRF analysis of vertebrate fossil bones, X-Ray Spectrometry 37, p. 293-297.

Paschalis, E. P., DiCarlo, E., Betts, F., Sherman, P., Mendelsohn, R. et Boskey, A. L., 1996 - FTIR Microspectroscopic Analysis of Human Osteonal Bone, Calcif Tissue Int 59, p. 480-487.

Piga, G., Malgosa, A., Thompson, T. J. U. et Enzo, S., 2008 - A new calibration of the XRD technique for the study of archaeological burned human remains, Journal of Archaeological Science 35-8, p. 2171-2178. 
Pucéat, E., Reynard, B. et Lécuyer, C., 2004 - Can crystallinity be used to determine the degree of chemical alteration of biogenic apatites?, Chemical Geology 205, p. 83-97.

Reiche, I., Chadefaux, C. et Müller, K., 2012 - Défis et méthodes de l'analyse des os archéologiques à micro- et nanoéchelle, ArchéoSciences, 35, ce volume.

Reiche, I., Favre-Quattropani, L., Calligaro, T. J. S., Bocherens, H., Charlet, L. et Menu, M., 1999 - Trace Element Composition of Archaeological Bones and Postmortem Alteration in the Burial Environment, Nuclear Instruments and Methods in Physics Research B 150, p. 656-662.

Reiche, I., Lebon, M., Chadefaux, C., Müller, K., Le Hô, A.-S., Gensch, M. et Schade, U., 2010. Microscale imaging of the state of preservation of 5000-years-old archaeological bones by synchrotron infrared microspectroscopy, Analytical and Bioanalytical Chemistry 394, p. 2491-2499.

Rey, C., Shimizu, M., Collins, B. et Glimcher, M.J., 1991 Resolution-enhanced Fourier Tranform Infrared Spectroscopy study of the Environment of Phosphate Ion in the Early Deposits of a solid Phase of Calcium Phosphate in Bone and Enamel and their Evolution with Age : 2. Investigations in the $v_{3} \mathrm{PO}_{4}$ Domain, Calcif Tissue Int 49, p. 383-388.

Sémah, F., Sémah, A.-M., Falguères, C., Gallet, X., Hameau, S., Moigne, A.-M. et SimanjuntaK, H. T., 2004 - The significance of the Punung karstic area (Eastern Java) for the chronology of the Javanese Palaeolithic, whit special reference to the Song Terus cave, Modern Quaternary Research in Southeast Asia 18, p. 45-62.

Shen, J., Fan, L., Yang, J., Shen, A. et Hu, J., 2010 - A longitudinal Raman microspectroscopic study of osteoporosis induced by spinal cord injury, Osteoporosis International 21, p. 81-87.
Stiner, M.C., Kuhn, S.L., Weiner, S. et Bar-Yosef, O., 1995 - Differential Burning Recrystallization, and Fragmentation of Archaeological Bone, Journal of Archaeological Science 22, p. 223-237.

Tavoso, A., 1987 - Le remplissage de la grotte Tournal à BizeMinervois (Aude), Cypsela, VI, Girona, p. 23-25.

Thomas, D. B., Fordyce, R. E., Frew, R. D. et Gordon, K. C., 2007 - A rapid, non-destructive method of detecting diagenetic alteration in fossil bone using Raman spectroscopy, Journal of Raman Spectroscopy 38, p. 1533-1537.

Timlin, J. A., Carden, A. et Morris, M. D., 1999 - Chemical Microstructure of Cortical Bone Probed by Raman Transects, Applied Spectroscopy 53, p. 1429-1435.

Trueman, C. N., Privat, K. et Field, J., 2008 - Why do crystallinity values fail to predict the extent of diagenetic alteration of bone mineral?, Palaeogeography, Palaeoclimatology, Palaeoecology 266, p. $160-167$.

Trueman, C. N. G., Behrensmeyer, A. K., Tuross, N. et Weiner, S., 2004 - Mineralogical and compositional changes in bones exposed on soil surfaces in Amboseli National Park, Kenya : diagenetic mechanisms and the role of sediment pore fluids, Journal of Archaeological Science 31, p. 721-739.

Weiner, S. et Bar-Yosef, O., 1990 - States of Preservation of Bones from Prehistoric Sites in the Near East: A Survey, Journal of Archaeological Science 17, p. 187-196.

Wess, T., Alberts, I., Hiller, J., Drakopoulos, M., Chamberlain, A. T. et Collins, M., 2002 - Microfocus Small Angle X-ray Scattering Reveals Structural Features in Archaeological Bone Samples; Detection of Changes in Bone Mineral Habit and Size, Calcified Tissue International 70, p. 103-110. 